\title{
International Journal of Physical Modelling in Geotechnics: Referees 2018
}

The following is a list of referees who have reviewed papers for International Journal of Physical Modelling in Geotechnics between 1 December 2017 and 30 November 2018. The Institution of Civil Engineers is very grateful for their assistance.

We are continually looking for suitable reviewers for papers submitted to International Journal of Physical Modelling in Geotechnics. Papers published in ICE journals must be submitted to at least two independent referees to judge accuracy, style, impact, importance and interest.

$\begin{array}{ll}\text { Christelle Abadie } & \text { Nilo Consoli } \\ \text { Osama Abuhajar } & \text { William Craig } \\ \text { Orestis Adamidis } & \text { Lijun Deng } \\ \text { Louis Akeh } & \text { Zong-Wei Deng } \\ \text { Hesham Aldaikh } & \text { Ali Derakhshani } \\ \text { Asad Al-Defae } & \text { Jelke Dijkstra } \\ \text { Marwan Alheib } & \text { Sam Divall } \\ \text { Márcio Almeida } & \text { Jean-Claude Dupla } \\ \text { Amin Askarinejad } & \text { Amr Ewais } \\ \text { Mahmoud Awad-Allah } & \text { Joerg Gattermann } \\ \text { Munwar Basha Basha } & \text { Kenneth Gavin } \\ \text { Richard Bathurst } & \text { Tsuyoshi Honda } \\ \text { Ryley Beddoe } & \text { Kenichi Horikoshi } \\ \text { Ryan Beemer } & \text { Ching-Chuan Huang } \\ \text { Leonora Begaj } & \text { Peter Hudacsek } \\ \text { Adam Bezuijen } & \text { Nejan Huvaj } \\ \text { Masoud Bonab } & \text { David Igoe } \\ \text { Alberto Bretschneider } & \text { Kazuya Itoh } \\ \text { Michael Brown } & \text { S.W. Jacobsz } \\ \text { Ana Carvalho } & \text { Ning-Jun Jiang } \\ \text { Mark Cassidy } & \text { Yujing Jiang } \\ \text { Shiao Huey Chow } & \text { Xin Kang } \\ \end{array}$

If you are interested in reviewing articles on any topic related to physical modelling in geotechnics, please submit your name, qualifications or CV, and areas of expertise. We are in need of individuals who will agree to review papers in a timely fashion (within 3 to 4 weeks of receipt) and provide confidential feedback to the Editorial Advisory Panel concerning the quality of the paper and any suggested revisions that would be appropriate.

If you are such a person, please contact Rebecca Rivers (tel.: +44 (0)207 665 2241; e-mail: rebecca.rivers@icepublishing.com) for more information on the referee process.

$\begin{array}{ll}\text { Mohammad Hossein Khosravi } & \text { Keshab Sharma } \\ \text { Masaki Kitazume } & \text { Brian Sheil } \\ \text { Rasmus Tofte Klinkvort } & \text { Paul Shepley } \\ \text { Sven Knutsson } & \text { Masahiro Shinoda } \\ \text { Junyoung Ko } & \text { Matias Silva Illanes } \\ \text { Junichi Koseki } & \text { Mark Stringer } \\ \text { Nozomu Kotake } & \text { Hiroko Suzuki } \\ \text { Jyant Kumar } & \text { W. Take } \\ \text { Chung-Jung Lee } & \text { Mark Talesnick } \\ \text { Srikanth Madabhushi } & \text { Anh Minh Tang } \\ \text { Alec Marshall } & \text { R.N. Taylor } \\ \text { Aminaton Marto } & \text { Luc Thorel } \\ \text { Kok Shien Ng } & \text { Tetsuo Tobita } \\ \text { Mitsu Okamura } & \text { Cristina Tsuha } \\ \text { Divya P. V. } & \text { Bo Vesterberg } \\ \text { Jean-Michel Pereira } & \text { Lei Wang } \\ \text { Raffaele Ragni } & \text { Shanyong Wang } \\ \text { Frédéric Rosquoet } & \text { David White } \\ \text { Ahmed Rouili } & \text { Daniel Wilson } \\ \text { Tomas Sabaliauskas } & \text { Xueliang Zhao } \\ \text { Fernando Saboya } & \text { Yewei Zheng } \\ \text { José Schiavon } & \text { Thomas Zimmie } \\ & \end{array}$

